$R=X / \sqrt{ } 3$ when $\alpha \gg 1$. $X$ has not been measured directly, but Pippard ${ }^{4}$ has obtained some indirect experimental evidence which indicates that $X$ does, in fact, exceed $R$ in the anomalous region.

The expression (2) has further been computed as a function of $\omega \tau$ for several values of the ratio $\alpha / \omega \tau$. $(\alpha / \omega \tau$ is independent of $\omega$ and, for a given metal, determines the value of $l$; the values chosen correspond to several temperatures between room temperature and liquid helium temperatures.) The ratios $R / R_{c l}$ and $X / X_{c l}$ are found to have the classical value unity for sufficiently low, and again for sufficiently high, frequencies (which are, however, well below the frequency for which the metal becomes transparent), and to pass through a maximum value at intermediate frequencies. The anomalous effects become less marked as the temperature increases, and have disappeared almost completely at room temperature. To give some idea of the orders of magnitude involved, choosing values of the parameters which correspond to a very pure specimen of silver at liquid helium temperatures, we find that $R / R_{c l}$ attains a maximum value of 180 at a wave-length of $69 \mu$, while $X / X_{c l}$ attains a maximum value of $5 \cdot 3$ at a wave-length of $7 \cdot 6 \mathrm{~cm}$. The absorption coefficient of the metal is increased in the same ratio as $R$; thus in the above example the theoretical absorption coefficient at $69 \mu$ is $1.44 \times 10^{-3}$, whereas the classical value is only $8 \times 10^{-6}$. Since the absorption is so small even in the anomalous case, an experimental verification of these predictions is likely to be very difficult.

A full account of the theory is to be published elsewhere.

Mathematics Department,

University of Manchester.

Trinity College,

Cambridge.

Nov. 19.

1 Pippard, A. B., Proc. Roy. Soc., A, 191, 385 (1947).

"Wilson, A. H., "The Theory of Metals", 158 (Cambridge, 1936).

"Titchmarsh, E. C., "Introduction to the Theory of Fourier Integrals" chap. 11 (Oxford, 1937).

4 Pippard, A. B., Proc. Roy. Soc., A, 191, 399 (1947).

\section{Use of 'Calyx' Burner to Determine Combustion Conditions}

Brinsley and Stephens ${ }^{1}$ have described an apparatus in which air and gas are pre-mixed and burned in a vessel from which the only exit is a small hole. When the proportion of air in a mixture originally air-rich is gradually reduced, the flame alters, and at one stage a violet sheath appears, surrounding the lower part of the flame. This sheath Brinsley and Stephens call a 'calyx'; they claim that it appears when the gas-air is that theoretically required for complete combustion.

It was thought that sampling the products of combustion as the theoretical mixture was burning would enable the maximum possible concentration of carbon dioxide in these products to be determined by a single analysis. This would be of service in testing gas appliances.

A mixture of coal gas and air was used. It was found that the flame is stable over only a small range of needle-valve settings : both the rate of supply of the mixture and its constitution have to be approximately correct or the flame goes out. Adjust-

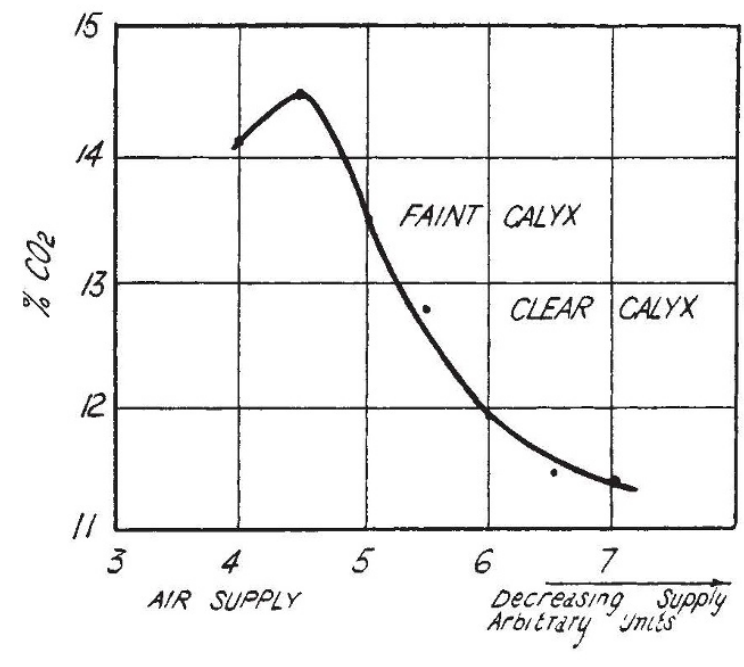

ment is not difficult once the operator has become familiar with the appearance of the flame in various circumstances, and the flame changes described by Brinsley and Stephens can then be readily observed.

Tests were made keeping the gas supply constant and cutting down the air in a series of arbitrary steps; at each setting a sample of the combustion products was taken and analysed for carbon dioxide. The results are shown in the accompanying graph. It will be seen that the calyx did not appear until after the maximum carbon dioxide concentration had been passed, and even then was only faintly visible. This reveals the chief objection to the use of the phenomenon for determination of theoretical combustion conditions. The calyx does not appear sufficiently suddenly; it 'fades in' as the air supply decreases, and the threshold visibility depends on the eyesight of the observer and on his experience. If it were used for determination of the maximum concentration of carbon dioxide in the combustion products, low results would be very probable.

Physics Division,

H. ROSTRON HINDLEY

Nash and Thompson, Ltd., Oakcroft Road,

Tolworth,

Surrey.

${ }^{1}$ Nature, 157, 622 (1946).

\section{A New Sulpha Compound ("6257") and its Use in Human Cholera Infection}

IT was observed by one of us (S. S. B.) in 1939 that hexa-methylene-tetra-amine, in a 10 per cent solution in normal saline, killed cholera vibrio in less than half an hour up to a concentration of $10^{12}$. Although interrupted by conditions of field service, efforts resulted in the production of three crude compounds of hexamine linkage to para-aminobenzene sulphonamide, experiments with which, on animal and human cholera infection, gave hope of successful chemotherapy against Asiatic cholera. A chance discussion with the scientific department of C.I.B.A. (Basle) resulted in our obtaining a condensation product-" 6257 " - of two molecules of $2 p$-aminobenzene sulphonamido-thiazole ('Cibazol') and three molecules of formaldehyde with the formula $\mathrm{C}_{21} \mathrm{H}_{22} \mathrm{O}_{6} \mathrm{~N}_{6} \mathrm{~S}_{4}$ (the actual constitution not having yet been worked out) and molar weight much greater 